\title{
Optical Feedback for Sensitivity Enhancement in Direct Raman Detection of Liquids
}

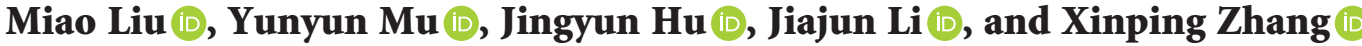 \\ Institute of Information Photonics Technology, Faculty of Science, Beijing University of Technology, Beijing 100124, China \\ Correspondence should be addressed to Xinping Zhang; zhangxinping@bjut.edu.cn
}

Received 27 February 2021; Accepted 30 April 2021; Published 10 May 2021

Academic Editor: Jau-Wern Chiou

Copyright (c) 2021 Miao Liu et al. This is an open access article distributed under the Creative Commons Attribution License, which permits unrestricted use, distribution, and reproduction in any medium, provided the original work is properly cited.

\begin{abstract}
Detection of low-concentration molecules in liquids has been a challenge in sensing technologies. Raman spectroscopy is an effective approach for trace detection, which is in fact a "volume-excitation" and "volume-collection" technique in the analysis of liquid samples. However, for the commonly employed one-pass excitation and back-scattering detection scheme, a large portion of both the excitation laser energy and the Raman-scattering light energy is wasted without efficient reuse or collection. In this consideration, we demonstrate a broadband optical feedback scheme by a curved high-reflection mirror for both the excitation and the Raman-scattering light, so that the excitation and the forward-propagating Raman signal can be back-reflected and collected with a high efficiency. Using the "F+2f" design, where $F$ and $f$ are the focal lengths of the focusing lens and curved reflection mirror, respectively, we were able to not only produce two focuses of the excitation laser beam but also extend the Raman interaction by a doubled distance. For the detection of pure ethanol molecules and the R6G molecules in water with a concentration of $10^{-3} \mathrm{M}$, the Raman signal was enhanced by a factor of about 5.6. The optical feedback scheme and discovered optical mechanisms supply effective improvements to the Raman spectroscopic measurements on liquid samples.
\end{abstract}

\section{Introduction}

Raman spectroscopy is one of the most powerful techniques for the identification of molecules by their "fingerprint" scattering spectrum $[1,2]$. It is a fast, simple, highly reproducible, and nondestructive detection tool, which can be utilized either quantitatively or qualitatively in the determination of low-concentration matters mixed with other molecules or dissolved in liquids [3-7]. This technique has been employed widely in the research fields of biochemistry and physics and in the various practical applications in the detection of specially interested substance $[6,8-10]$. However, Raman-scattering signal is very weak, which has an intensity lower than $\left(10^{-6}\right)$ that of the excitation. Therefore, approaches for enhancing the Raman-scattering signals are always important for improving and realizing the related detection techniques [11-13].

For liquid samples, due to the lower molecular concentration and the mobile form of the molecular substances, the signal intensity is even lower, introducing more challenges for the sensitive and reliable detections [14-17]. One of the most typical examples is the detection of pollutant molecules in environmental or drinking water. In particular, the interaction between the focused laser beam and the liquid samples has a volume extending nearly symmetrically about the focus in opposite directions, implying that forward and backward propagation Raman-scattering signals should be collected for the whole excitation volume. However, in most cases, the Raman-scattering light is collected in the forward or backward direction with respect to the direction of the excitation light beam that is focused into the target sample. Thus, only a portion of the back- or forward-scattering light is collected in most of the Raman spectroscopic measurements, reducing largely the intensity of the Raman-scattering light and leading to largely reduced signal-to-noise (SNR) ratio and the detection sensitivity. Many designs have been reported for improving the Raman detection technique [18-20]. These approaches may include single-channel multiplex optrode $[21,22]$, liquid core waveguides [23, 24], and fiber probes [25]. Parabolic reflection mirror and multipass schemes have been 
reported for the Raman spectroscopic systems, which enabled large enhancement of the detection sensitivity $[12,13,18,19]$.

In this work, we first verify the volume-excitation and volume-collection nature of the Raman-scattering spectroscopy experimentally and then present designs of optical feedback schemes for collecting the forward-propagating Raman-scattering light using flat and curved reflection mirrors. Using a retro-reflection design by curved mirrors with an "F+2f" scheme, we achieved not only the doubled focusing of the excitation laser beam but also the doubled collection of the Raman signal by the optical feedback. Thus, we realized an enhancement factor of 5.6 in the Raman spectroscopic measurement on ethanol. Compared with the reported methods $[12,13,18,19]$, our " $F+2 f$ " scheme has a simplest design and low-cost configuration, which produces even higher enhancement. Although we employed pure ethanol and R6G in water as the analytes in this experimental work, the most important motivation is to find effective and practical approaches for improving the sensitivity of Raman spectroscopy, which can be potentially utilized in the direct detection of pollutant molecules in environmental water.

\section{Optical Design for the Enhancement of the Volume Raman Spectroscopy}

Figure 1 shows the geometry for the optical design of the Raman spectroscopic measurements on liquid samples of pure ethanol. We used a fiber-coupled $785 \mathrm{~nm}$ laser as the excitation, which was recollimated after being coupled out of the fiber. After passing through a band-pass filter for $785 \mathrm{~nm}$, the laser beam was reflected by a high-reflection (HR) mirror and a dichroic mirror for $785 \mathrm{~nm}$ high-reflection and longerwavelength transmission. Then, the narrow-band $785 \mathrm{~nm}$ laser beam was focused into the liquid sample. The Ramanscattering light was collected by the focusing lens to pass through the dichroic mirror and the long-pass filter, so that the excitation laser at $785 \mathrm{~nm}$ was "blocked" through high reflection by the dichroic mirror and the long-path filter. Thus, only the Raman-scattering light reached the focusing lens for coupling into the detection optical fiber of the Raman spectrometer. The Raman spectrum can be then detected and displayed. What is particular for our design is that a gold mirror is located at varied depth inside the liquid sample along the axis of the detection path to supply optical feedback for both the excitation and scattering light, as shown in Figure 1. Flat and curved gold-coated mirrors have been employed in the experiments, so that different optical feedback mechanisms can be investigated and compared. The continuous-wave excitation laser at $785 \mathrm{~nm}$ was purchased from Shanghai Oceanhood Optoelectronics Tech Co., LTD, which supplies a maximum power of $300 \mathrm{~mW}$; however, we used only $125 \mathrm{~mW}$ in all of the experiments. A Raman spectrometer QE-PO1609 from Ocean Optics was equipped with the system, where the software is provided by the supplier and a collection integration time of $3 \mathrm{~s}$ was used in all of our measurements.

The principles for the feedback-enhanced Raman spectroscopy can be understood by analyzing the geometry in the dashed circles in Figure 1. Flat and curved mirrors with gold coatings are employed, since gold films have high reflection for both the excitation and Raman-scattering light in the range from 785 to about $850 \mathrm{~nm}$. It needs to be stressed here that the gold coatings simply supply broadband high reflections, and no surface-enhanced Raman-scattering (SERS) effect is involved here. It is apparent that although a flat feedback mirror can also reflect a large portion of the forward-propagating Raman signals back to the detection path, only a limited fraction can be coupled back into the detector. This is due to the large divergence of both the excitation and Raman-scattering light, where the feedback flat mirror has to be placed below the focus of the excitation laser beam to ensure reasonable focusing position and sufficiently long interaction distance. Thus, the reflection path is different from the back-reflection of the excitation and there is a limited coupling efficiency for the feedback. Furthermore, a flat mirror cannot supply additional focuses to intensify or extend the length of the Raman interactions.

In contrast, using a curved mirror with a focal length of $f$ to replace the flat mirror, we can improve the configuration largely in two aspects: (1) If the curved mirror is placed below the focus at a distance of $2 f$, precise retro-reflection can be achieved for the excitation laser beam. Thus, the excitation laser beam is focused twice at the same location; meanwhile, the intensive interaction length is doubled. (2) The Raman-scattering light propagating in the forward direction can be back-reflected and collected sufficiently by the detection optical systems. Thus, the detected Raman signal can be enhanced at least by a factor of 8 . However, due to the difficulties in the management and adjustment of the curved mirror below liquids, the alignment of the optical axis of the curved mirror with that of the excitation and detection light path, and the precise location of its position with respect to the laser focus, the enhancement factor cannot be utilized to its full value. In our experiments in this work, we used pure ethanol as the liquid sample, and an enhancement factor of about 5.6 has been achieved.

\section{Results and Discussions}

\subsection{Verification of Volume-Excitation Volume-Collection} Performance. To demonstrate the volume-excitation volume-collection feature of the Raman spectroscopic measurements on liquids, we carried out a verification experiment, as illustrated in the inset of Figure 2(b). A goldcoated flat mirror was placed in the liquid sample with a tilting angle of $45^{\circ}$, which functions as a blocker of the excitation laser beam. Due to this large tilting angle, no reflection by the mirror of the Raman signal can be collected by the detection system. Thus, moving this tilted mirror up and down along the optical axis accomplishes the adjustment of the interaction distance. It needs to be noted that ethanol has a refractive index of about 1.362 , which leads to the extension of the focal length below the liquid surface. Additionally, in all of our experiments below, the depth $z$ has been measured outside the glass container (a $100 \mathrm{ml}$ beaker), which is an absolute distance and can be used without modification by the refractive index of ethanol. 


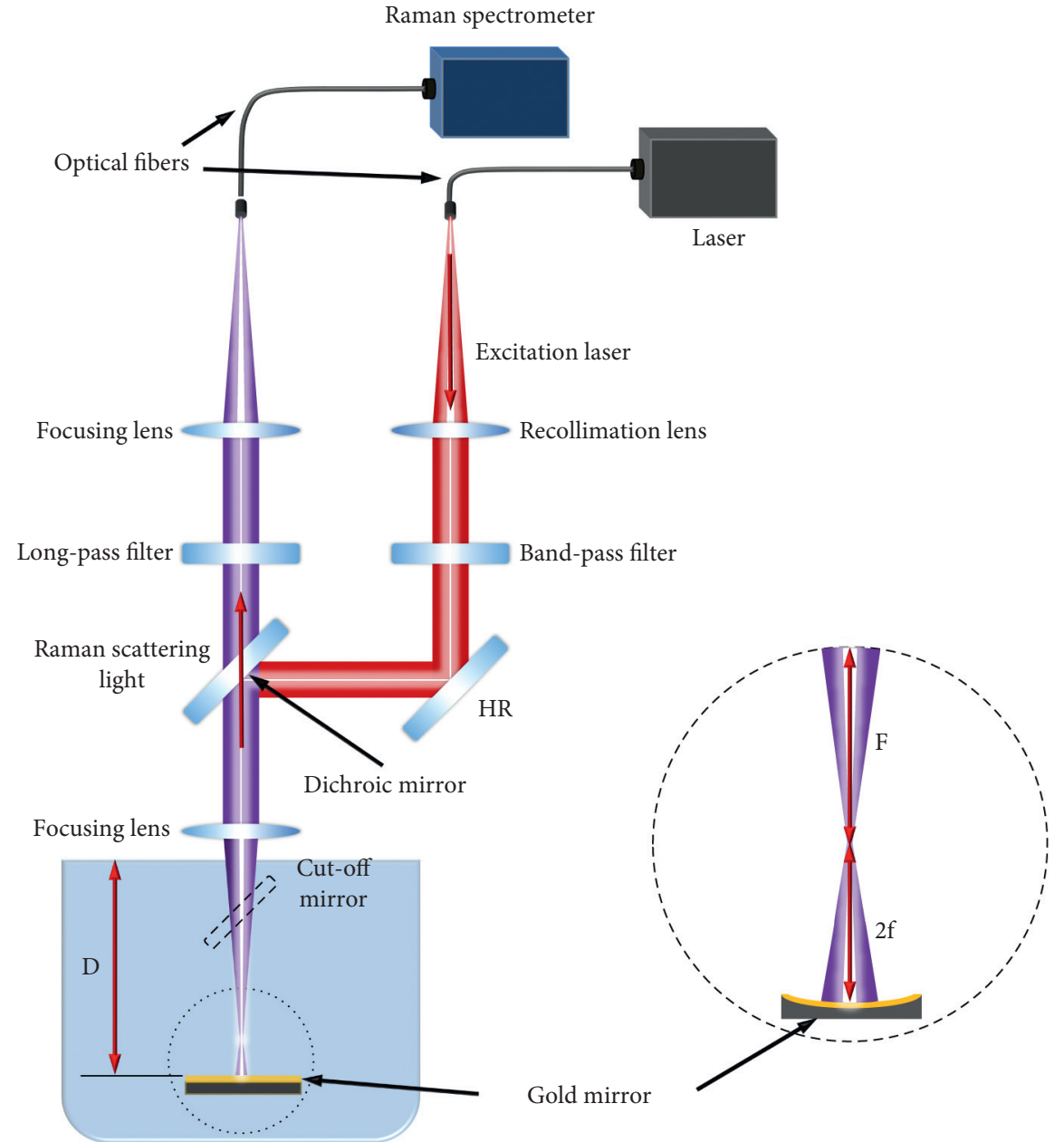

FIGURE 1: Experimental setup for optical feedback-enhanced Raman spectroscopic measurements on liquid samples.

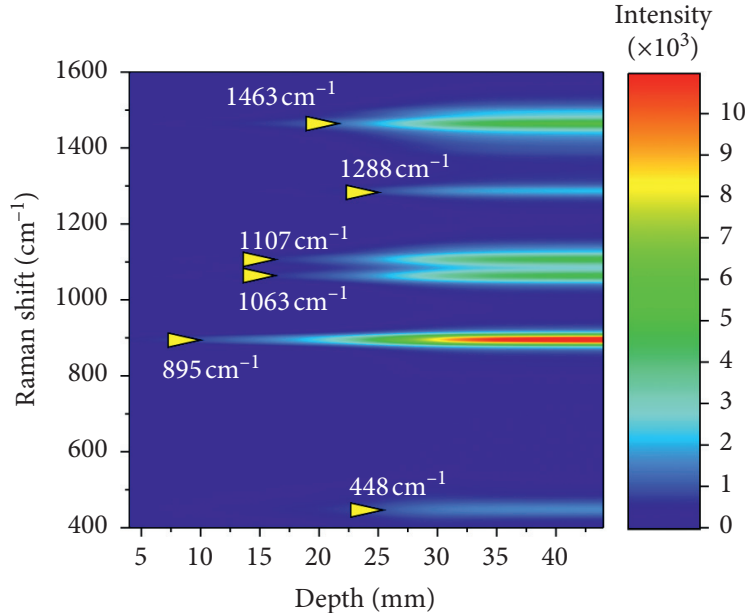

(a)

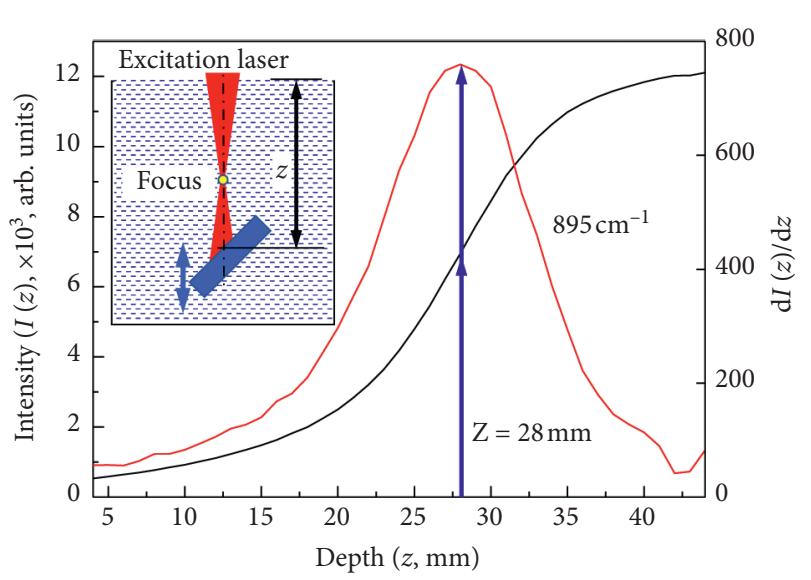

(b)

FIGURE 2: (a) Intensity of the Raman-scattering spectrum as a function of Raman shift and depth of the "blocking" mirror below the liquid surface, where 6 Raman peaks can be observed. (b) The Raman signal peak intensity $(I(z))$ at about $895 \mathrm{~cm}^{-1}$ varying with the depth $(z)$ of the "blocking" mirror (black curve). Red curve: $\mathrm{d} I(z) / \mathrm{d}(\mathrm{z})$, showing an inflection point roughly at the focus of the laser beam with $z=28 \mathrm{~mm}$. Inset: schematic illustration of the experimental setup. 
Figure 2(a) shows a three-dimensional (3D) plot of the Raman signal intensity as a function of Raman shift and the depth of the location of the tilted mirror with respect to the liquid/air interface, which was recorded by the position of the excitation laser spot on the surface of the tilted mirror. Clearly, six Raman peaks can be observed at about 448, 895, $1063,1107,1288$, and $1463 \mathrm{~cm}^{-1}$, which are typical of ethanol solution. Among them, we can observe a strongest signal at about $895 \mathrm{~cm}^{-1}$ and pick up this peak for our analysis on the enhancement effect. We have used a focusing lens with a focal length of $25.4 \mathrm{~mm}(F=25.4 \mathrm{~mm})$ and tuned the depth of the tilted mirror from 4 to $44 \mathrm{~mm}$ under the top surface of the ethanol solution in a beaker with a volume of $100 \mathrm{~mL}$. As the excitation laser beam is focused into the liquid, the focal length may be largely extended due to the refraction by the liquid with larger refractive index than air. This explains why the focus is observed at about $28 \mathrm{~mm}$ by depth inside the liquid, as shown in Figure 2(b), which does not include the distance above the liquid surface. The black curve in Figure 2(b) shows the variation of the Raman signal intensity with depth inside the liquid. Clearly, the intensity increases dramatically before reaching the focus; then, the increasing rate is slowed down, and a saturation effect can be observed with $z>32 \mathrm{~mm}$. A turning point is observed at $z=28 \mathrm{~mm}$, which is exactly around the focus of the excitation laser beam and can be verified by the calculation of $\mathrm{d} I(z) / \mathrm{d} z$, as shown by the red curve in Figure 2(b). This increasing speed curve is almost symmetrical about the focus of the laser beam. According to Figure 2(b), for $z>44 \mathrm{~mm}$, the Raman signal stays nearly constant (black curve) and the differential curve (red curve) reaches almost 0 again, implying that the interaction beyond $44 \mathrm{~mm}$ does not make any contribution to the Raman signal any more. Therefore, the interaction length that is required for achieving efficient Raman signal is even larger than $44 \mathrm{~mm}$, which is nearly double the focal length of the focusing lens. Thus, the experimental results in Figure 2 verify the volume-excitation and volume-collection nature of the Raman spectroscopic measurement on liquids. This implies not only a required sufficiently long interaction distance for the excitation laser with the sample but also the importance of optical feedback in the collection scheme of the Raman signal.

\subsection{Optical Feedback Using a Planar Reflector. A straight-} forward solution for the optical feedback is using a flat reflection mirror, which reflects the forward-propagating Raman signals back to the detection optical path and enhances the detected signal intensity, as shown in Figure 3. The inset of Figure 3(b) illustrates schematically the experimental setup, where a gold-coated flat mirror was placed inside the liquid sample and moved up and down to optimize the Raman signal. An excitation laser power of $125 \mathrm{~mW}$ and an integration time of $3 \mathrm{~s}$ have been employed in the measurements.

In Figure 3(a), the Raman spectrum is recorded as a function of the depth of the flat reflector. Again, multiple Raman peaks can be observed and the most intensive one is located at about $895 \mathrm{~cm}^{-1}$. However, the strongest Raman signal is observed at a depth of the optical feedback mirror of about $z=34 \mathrm{~mm}$. Figure $3(\mathrm{~b})$ shows the measured intensity of the Raman signal at $895 \mathrm{~cm}^{-1}$ which varies with value of $z$. The red line in Figure 3(b) marked the highest intensity of the Raman signal measured without optical feedback by the flat reflector, which is about $11.8 \times 10^{3}$. With the optical feedback by the flat reflector, the peak intensity of the Raman signal is measured to be $34 \times 10^{3}$ at $z=34 \mathrm{~mm}$, implying an enhancement factor of about 2.88, as highlighted in Figure 3(b).

It needs to be noted that if the flat reflector is above the focus of the laser beam, the practical focus inside the liquid sample might be reflected out of the liquid, so that the Raman signal is very small at the beginning and the increase of it is slow, as shown by the downward arrow for $z<12 \mathrm{~mm}$. As the focus enters the liquids, the increase of the Raman signal intensity becomes much faster, as can be seen on the black curve with $z>12 \mathrm{~mm}$. A turning point can be observed when the focus of the excitation laser beam reaches the surface of the liquid, which corresponds to the first peak of the blue curve plotted for $d I(z) / d z$, as shown in Figure 3(b) and highlighted by the upward solid blue arrow. With lowering the flat reflector further, the Raman signal increases quickly and reaches a second turning point at about $z=28 \mathrm{~mm}$, as highlighted by the dashed upward blue arrow in Figure 3(b) for the blue curve. This turning point agrees with that in Figure 2(b). With lowering the flat reflector further, we will observe the peak intensity of the Raman signal at about $z=34 \mathrm{~mm}$ with $d I(z) / d z=0$.

If we examine the enhancement effect by a flat reflection mirror as the optical feedback, we may find that the configuration can be improved in two aspects by replacing the flat reflection mirror with a curved one. (1) A second focus of the excitation laser with nearly the same size, the same location, and the same intensity may be produced in the back-reflected beam; thus, the enhancement factor may be doubled $(2.88 \times 2=5.76)$. (2) Since the flat mirror reflected a divergent beam back to the detection light path, the feedback beam size is larger than the effective aperture of the focusing/collection optics, producing a collection efficiency of roughly $(25 / 34)^{2} \approx 54 \%$. If such a loss is compensated by a curved reflection mirror and enhancement factor can be estimated as $2.88 \times 2 / 54 \% \approx 10.6$, which is nevertheless a theoretical estimation, we should also take into account the light intensity distribution over the transverse mode of the Raman-scattering light beam and the size of the curve mirror with respect to that of the excitation and scattering light beams. Even with this consideration, an enhancement factor larger than 8 is a reasonable estimation.

3.3. Optical Feedback Using a Curved Reflector. Based on above discussions, a more efficient optical feedback is using a curve mirror with a concave reflection surface, as shown in Figure 1 and in the inset of Figure 4. In this design, we used a curved mirror with a diameter of $12.7 \mathrm{~mm}$, where the spherical reflection surface has a curvature radius of about $10 \mathrm{~mm}$, corresponding to a focal length of $5 \mathrm{~mm}$. The curved 


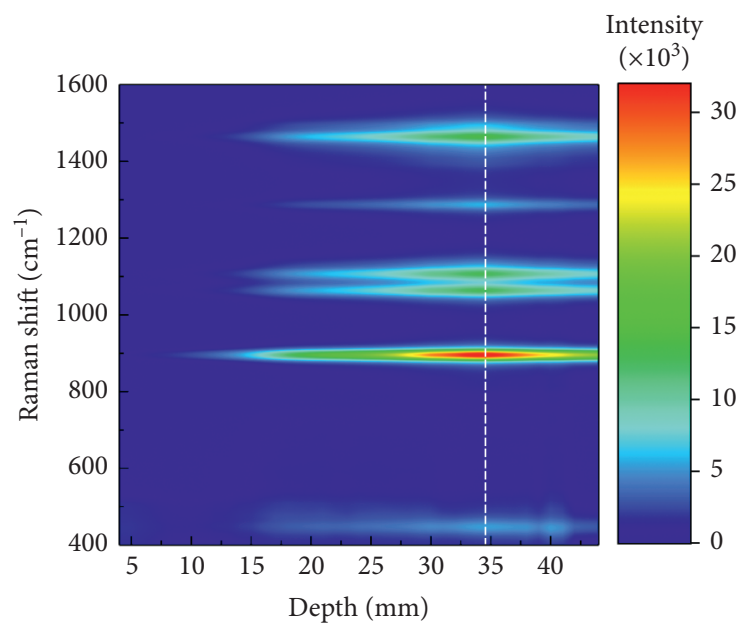

(a)

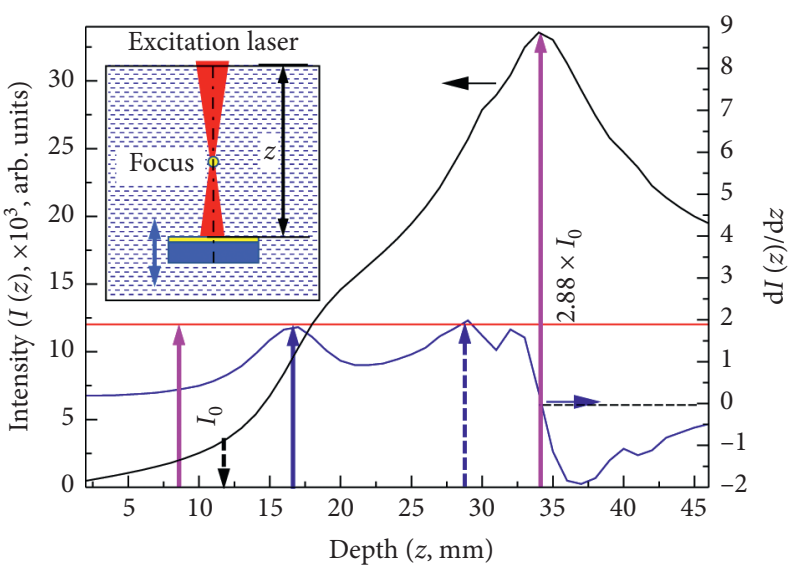

(b)

Figure 3: (a) The Raman signal intensity as a function of Raman shift and the location depth of the flat feedback mirror underneath the liquid surface. (b) The Raman signal peak intensity $I(z)$ at $895 \mathrm{~cm}^{-1}$ at different depths $(z)$ of the flat optical feedback mirror. Blue curve: calculation of $d I(z) / d(z)$. Inset: schematic illustration of the measurement setup.

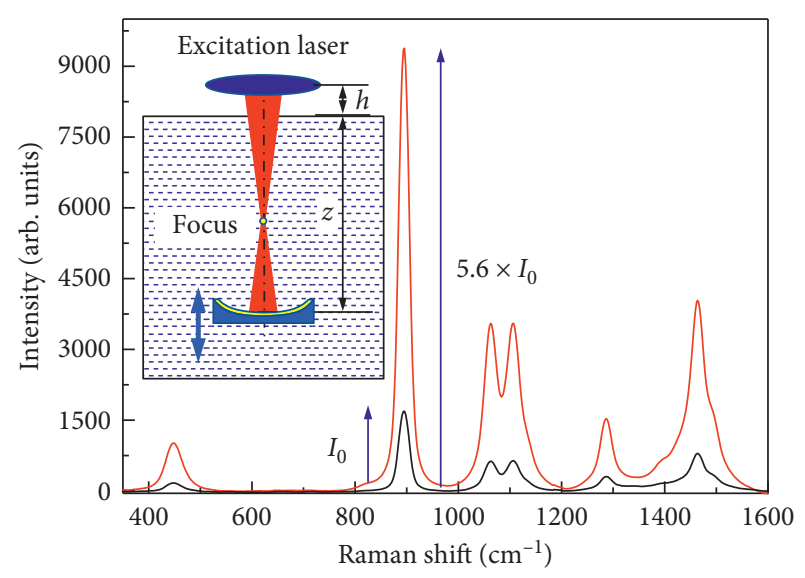

FIGURE 4: Raman-scattering spectra measured with (red) and without (black) a curved optical feedback mirror. Inset: schematic illustration of the experimental setup.

surface was coated with gold for high reflection at both the excitation and Raman-scattering wavelengths. The parameters of the curved mirror have been optimized not only through obtaining the largest enhancement factor of the Raman signals but also on the basis of the allowed location depth underneath the liquid surface, the excitation laser beam size, the dimensions of other optical and mechanical elements, and the size/volume of the liquid container. This not only supplies more efficient collection of the Raman signal and possibly optimized mode matching between feedback Raman signal beam and the collection optics but also enables a new focus of the excitation lasers, which doubles the whole Raman-scattering process. Thus, the enhancement factor demonstrated in Figure 3(b) may be further increased to about 5.6, as shown in Figure 4 .
In Figure 4, we plot the Raman spectra that are measured with (red) and without (black) the feedback mirror. A concave mirror with its curve surface coated with gold was employed as the optical feedback reflector, which has high reflection for both the excitation $(785 \mathrm{~nm})$ and Ramanscattering wavelength $(810 \sim 900 \mathrm{~nm})$. If assuming a focal length of $F$ for the external focusing lens and $f$ for the curved mirror, the optimized depth of the curve mirror should be written as $z \approx F-h+2 f$. Since we have $h \approx 2 \mathrm{~mm}, F=25.4 \mathrm{~mm}$, and $f=5 \mathrm{~mm}$, we may estimate approximately $z \approx 33.4 \mathrm{~mm}$. However, we need to be aware of the modification of $F$ by the refraction into ethanol, the value of $z$ can be extended, and we have roughly $z \approx 44 \mathrm{~mm}$. Thus, the first focus produced by the external focusing lens is imaged by the curved mirror on its original position, forming a second focus for Raman excitation. Moreover, in such a geometry, the collected Raman signal by the feedback curved mirror is reflected back to the collection optics with the best mode matching, ensuring the highest coupling efficiency. However, there are more challenges in the optical alignment with such a curved feedback mirror. Because there are more sensitive parameters in such a geometry, the variation of the Raman signal with adjusting the depth $z$ is not as smooth as with a flat mirror, since a slight tilting or horizontal move of the curved mirror may change largely the Raman signal, where the optical axis of the curved mirror has to be aligned precisely with that of the excitation laser beam. On such basis, we have achieved the best enhancement factor of 5.6 by optimizing the three-dimensional positions and the tilting angle of the curve mirror.

In fact, the experimental results in Figure 4 can still be largely improved in multifold aspects: (1) The alignment of the axis of the feedback curved mirror with that of the collection optics is very critical, where even a slight 
misalignment may lead to dramatic reduction in the detection Raman signal. Even with very careful adjustment, the signal has not been optimized to its best value. This is mainly due to the optical design for lowering and adjusting the curved mirror in the liquid sample, where a long mechanical arm has to be used to send the curved mirror into a depth inside the liquid sample. (2) Optimization of the focal lengths of both the external focusing lens and the curve mirror can improve the excitation and collection efficiency further. (3) Enlargement of the diameter of the curve mirror may increase the collection area largely and facilitate the collection to a much improved extent. (4) Coating with gold is not a best solution to achieve strong optical feedback, where gold still has much absorption and there is large variation in the reflection rate over the studied spectral band. Using broadband dielectric coatings with a high-reflection rate may improve the enhancement factor further remarkably.

3.4. Detection of R6G Molecules in Water. In the above experiments, we used pure ethanol as our detection sample; however, this does not mean that our design and photophysical mechanisms apply only to pure liquid samples. This is mainly because pure ethanol provides conveniently a typical sample for revealing the "volume-excitation and volume-collection" mechanism and further the importance of the "optical feedback" mechanisms. Moreover, ethanol is not a harmful liquid and it has very typical Raman peaks suitable for most of the excitation lasers and for most of the Raman spectrometers.

However, one of the most important motivations of this work is to supply basis for the development of practical systems potentially for the detection of pollutant molecules in water. Therefore, Raman spectroscopic measurement on molecules in aqueous solvent is of critical importance. For such a purpose, we prepared an aqueous solution of R6G with a concentration of $10^{-3} \mathrm{~mol} / \mathrm{L}$ and carried out measurement using the detection system with and without the optical feedback design. In the determination of the lower limit of the concentration of the sample solution, we should be able to observe clear Raman signals without the optical feedback, so that the enhancement factor by the optical feedback scheme can be evaluated precisely. Without the optical feedback, we were not able to find any Raman signals at an R6G/water concentration of $10^{-4} \mathrm{~mol} / \mathrm{L}$, which is the reason why we selected a concentration of $10^{-3} \mathrm{~mol} / \mathrm{L}$ in the experiment.

Figure 5 shows the measurement results, where the red and black curves correspond to the Raman spectra measured with and without the optical feedback, respectively. A comparison between the amplitudes of the two Raman spectra resolves an enhancement factor of about 5.6 at $1379 \mathrm{~cm}^{-1}$, which is the largest Raman signal for R6G. Such an excellent reproducibility of the enhancement factor confirms the optical mechanisms and the effectiveness of the optical feedback scheme. Moreover, this result demonstrates stability and independence on the liquids of the optical feedback mechanisms.

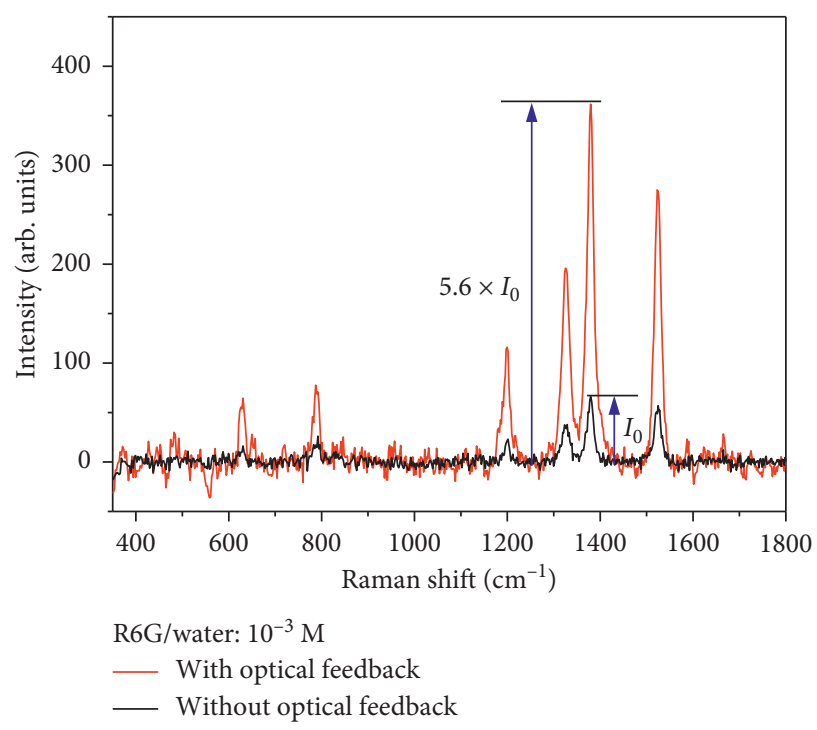

FIGURE 5: Raman-scattering spectra measured on R6G/water solution with a concentration of $10^{-3} \mathrm{Mol} / \mathrm{L}$ using a system with (red) and without (black) an " $F+2 f$ " optical feedback scheme.

\section{Conclusions}

We report designs of optical feedback schemes for the signal enhancement of Raman-scattering spectroscopy. Experiments demonstrate volume-excitation and volume-collection features of the Raman detection of liquids and verify the strong forward-propagating Raman-scattering signals. We thus conclude the necessity and importance of incorporating optical feedback schemes into the detection system. Flat and curved back-reflectors have been employed in investigating the optical feedback mechanisms, which lead to enhancement factors of 2.88 and 5.6 for the Raman-scattering signals, respectively. Curved mirrors with an " $F+2 f$ " scheme for precise retro-reflective imaging of the forward-propagating light beams are verified to be the best optical feedback design, which not only produces an additional focus of the excitation laser beam but also enables most efficient collection of the Raman-scattering signals with perfect mode matching in the back-reflection coupling. The revealed optical mechanisms and the presented designs are important for solving the challenges in the detection of low-concentration molecules in liquids. We summarize some further remarkable progresses made in this work as follows: (1) We develop systematic modeling on the variation of the Raman spectroscopic response with liquid depth, which not only supplies deep insights into the photophysics involved in Raman spectroscopy but also provides aided guidance for the design of Raman detection system, in particular for direct detection in liquids. (2) Our optical feedback scheme facilitates a potentially convenient and suitable design for the in situ detection of pollutant molecules in environmental water by Raman spectroscopy. (3) The design and the developed system are simple, practical, and efficient, as compared with the previously reported approaches. (4) Our design can be easily integrated with the Raman detection 
system, in particular with the fiber-based detection head, for direct in situ Raman detection in environmental water.

\section{Data Availability}

The data used to support the findings of this study are included within the article.

\section{Conflicts of Interest}

The authors declare that they have no conflicts of interest.

\section{Acknowledgments}

The authors acknowledge the National Natural Science Foundation of China (61735002 and 12074020) and the Beijing Municipal Education Commission (KZ202010005002).

\section{References}

[1] C. Wang, L. H. Zeng, and Z. Li, "Review of optical fibre probes for enhanced Raman sensing," Journal of Raman Spectroscopy, vol. 48, no. 8, pp. 1040-1055, 1999.

[2] R. R. Jones, D. C. Hooper, L. W. Zhang, D. Wolverson, and V. K. Valev, "Raman techniques: fundamentals and frontiers," Nanoscale Research Letters, vol. 14, p. 231, 2019.

[3] E. K. Oztekin, D. J. Burton, and D. W. Hahn, "Detection of explosives using differential laser-induced perturbation spectroscopy with a Raman-based probe," Applied Spectroscopy, vol. 70, no. 4, pp. 676-687, 2016.

[4] T. Lima, M. Musso, and D. Menezes, "Using Raman spectroscopy and an exponential equation approach to detect adulteration of olive oil with rapeseed and corn oil," Food Chemistry, vol. 333, Article ID 127454, 2020.

[5] D. P. Aykas, M.-L. Shotts, and L. E. Rodriguez-Saona, “Authentication of commercial honeys based on Raman fingerprinting and pattern recognition analysis," Food Control, vol. 117, Article ID 107346, 2020.

[6] D. V. Petrov, "Raman spectrum of ethane in methane environment," Journal of Raman Spectroscopy, vol. 49, no. 4, pp. 771-774, 2018.

[7] M. M. Zhang, Y. N. Guo, F. Hu, Z. Liu, J. L. Shi, and X. D. He, "Research on marine oil spill pollution detection based on laser Raman spectroscopy," Second Target Recognition and Artificial Intelligence Summit Forum, vol. 114, 2020.

[8] D. W. Shipp, F. Sinjab, and I. Notingher, "Raman spectroscopy: techniques and applications in the life sciences," Advances in Optics and Photonics, vol. 9, no. 2, pp. 315-428, 2017.

[9] M. M. Zhang, Y. N. Guo, F. Hu, Z. Liu, J. L. Shi, and X. D. He, "Research on marine oil spill pollution detection based on laser Raman spectroscopy," SPIE Newsroom, 2020.

[10] H. He, D.-W. Sun, H. Pu, L. Chen, and L. Lin, "Applications of Raman spectroscopic techniques for quality and safety evaluation of milk: a review of recent developments," Critical Reviews in Food Science and Nutrition, vol. 59, no. 5, pp. 770-793, 2019.

[11] J. Wang, K. Liu, S. Jin, L. Jiang, and P. Liang, "A review of Chinese Raman spectroscopy research over the past twenty years," Applied Spectroscopy, vol. 74, no. 2, pp. 130-159, 2020.

[12] A. Yu, D. Zuo, and X. Wang, "Optimization of parabolic cell for gas Raman analysis," Journal of Raman Spectroscopy, vol. 50, no. 5, pp. 731-740, 2019.
[13] Q. Liu, M. S. Schmidt, H. Thienpont, and H. Ottevaere, "Compact conical beam shaper and freeform segmented reflector for SERS analysis," Optics Express, vol. 28, no. 11, pp. 16163-16174, 2020.

[14] X.-X. Zhou, R. Liu, L.-T. Hao, and J.-F. Liu, "Identification of polystyrene nanoplastics using surface enhanced Raman spectroscopy," Talanta, vol. 221, Article ID 121552, 2021.

[15] X. Zhang, Z. Du, Z. Luan et al., "In situ Raman detection of gas hydrates exposed on the seafloor of the south China sea," Geochemistry, Geophysics, Geosystems, vol. 18, no. 10, pp. 3700-3713, 2017.

[16] L. Li, Z. Du, X. Zhang et al., "In situ Raman spectral characteristics of carbon dioxide in a deep-sea simulator of extreme environments reaching $300{ }^{\circ} \mathrm{C}$ and $30 \mathrm{MPa}$," Applied Spectroscopy, vol. 72, no. 1, pp. 48-59, 2018.

[17] J. Shan, Y. Zhang, J. Wang, T. Ren, M. Jin, and X. Wang, "Microextraction based on microplastic followed by SERS for on-site detection of hydrophobic organic contaminants, an indicator of seawater pollution," Journal of Hazardous Materials, vol. 400, Article ID 123202, 2020.

[18] Y. Tian, J. W. Su, J. Ju, and Q. Liu, "Efficiency enhancement of Raman spectroscopy at long working distance by parabolic reflector," Biomedical Optics Express, vol. 8, no. 11, pp. 5243-5252, 2017.

[19] D. Zhang, X. Wang, K. Braun et al., "Parabolic mirror-assisted tip-enhanced spectroscopic imaging for non-transparent materials," Journal of Raman Spectroscopy, vol. 40, no. 10, pp. 1371-1376, 2009.

[20] Q. Liu, G. Barbieri, H. Thienpont, and H. Ottevaere, "Integrated confocal Raman probe combined with a free-form reflector based lab-on-chip," SPIE Newsroom, vol. 103, 2017.

[21] Q. Giuseppe, Z. Gianluigi, and M. Stefano, "Nanosphere lithography on fiber: towards engineered lab-on-fiber SERS optrodes,” Sensors, vol. 18, no. 3, p. 680, 2018.

[22] F. Galeotti, M. Pisco, and A. Cusano, "Self-assembly on optical fibers: a powerful nanofabrication tool for next generation "lab-on-fiber" optrodes," Nanoscale, vol. 10, no. 48, pp. 22673-22700, 2018.

[23] C. Wang, Y. Xu, R. Wang et al., "Fabrication and effect study of microfluidic SERS chip with integrated surface liquid core optical waveguide modified with nano gold," Microsystem Technologies, vol. 23, no. 8, pp. 3059-3068, 2017.

[24] P. Measor, L. Seballos, D. Yin et al., "On-chip surface-enhanced Raman scattering detection using integrated liquidcore waveguides," Applied Physics Letters, vol. 90, no. 21, Article ID 211107, 2007.

[25] F. Antonino, D. Cristiano, and B. Francesco, "A shapeengineered surface-enhanced Raman scattering optical fiber sensor working from the visible to the near-infrared," Plasmonics, vol. 8, no. 1, pp. 13-23, 2013. 